\title{
Exercise-Induced Hypertension: A Review of Plausible Mechanisms and Clinical Significance
}

\author{
Ayechew Adera Getu*
}

\begin{abstract}
This literature review is aimed to present the effect of exercise on blood pressure. More specifically it addresses the pathophysiological mechanisms as to how exaggerated blood pressure is occurring following experimental exercise and its predictive power for future new onset hypertension. Literatures searched using key words; 'Cardiovascular diseases', 'Exercise', 'hypertension', 'Hypertensive Response to Exercise (HRE)', 'Hemodynamic Changes' from search engines including PubMed, Cochrane Database and Google scholar. Blood pressure (BP) is the lateral pressure exerted on the wall of elastic arteries and it is critical for distribution of blood to metabolic tissues in need of oxygen and nutrients. Elevated BP or hypertension is often considered as a silent killer and is a risk factor for cardiac, neuronal and renal insults. A systolic BP of $\geq 210 \mathrm{mmHg}$ in males and $\geq 190 \mathrm{mmHg}$ in females to exercise testing is defined the term exercise-induced hypertension. An exaggerated increase in BP to exercise is a good predictor for the incidence of hypertension in other wise normotensive individuals. A hyperactivity of the sympathetic nervous system along with activation of renin-angiotensin system, a pre-existed endothelial dysfunction associated with a defect in release of nitric oxide, arterial stiffness and concurrent metabolic syndrome are the putative factors involved in exaggerated BP response to exercise. An exaggerated BP response to exercise in normotensives is a predictor for new onset hypertension and target organ damage. Therefore, early detection is paramount to prevent the complication and economic cost of hypertension.
\end{abstract}

Key words: Cardiovascular Disease, Exercise, Hypertension, Hypertensive Response to Exercise, Hemodynamic Changes.

\section{INTRODUCTION}

Blood pressure (BP) is the lateral pressure exerted on the wall of blood vessels and is a homeostatic parameter critical for distribution of blood to metabolic tissues. The mean arterial blood pressure is responsible for tissue perfusion throughout the cardiac cycle (i.e. during contraction and relaxation of the ventricle). The Joint National Committee on Prevention, Detection, Evaluation and Treatment of High Blood Pressure in its seventh report ${ }^{[1]}$ provides a classification of blood pressure for adults aged $\geq 18$ years. Normal BP is defined as levels $<120 / 80 \mathrm{mmHg}$. Systolic blood pressure (SBP) of $120-139 \mathrm{mmHg}$ or diastolic blood pressure (DBP) of $80-89 \mathrm{mmHg}$ is classified as prehypertension. Individuals in prehypertension state are at high risk of progression to hypertension. Hypertension is defined as systolic blood pressure $\geq 140 \mathrm{mmHg}$ or diastolic blood pressure $\geq 90 \mathrm{mmHg}$. Hypertension is a key risk factor for cardiovascular, neuronal and renal diseases. ${ }^{[2]}$ It is often a silent medical condition and becoming the major cause of premature death affecting about 1.13 billion people worldwide in which the majority of cases are living in low- and middle-income countries. ${ }^{[3]}$

\section{Determinants of Blood Pressure}

Blood pressure is the result of the peripheral arterial resistance and the cardiac out. The cardiac output is the volume of blood that is ejected from the ventricles in a minute. Blood pressure normally fluctuates in a single cardiac cycle (i.e. increases during the ejection process (ventricular systole) and decreases during the filling process (ventricular diastole). According to Hagen - Poiseuille law, blood pressure depends on cardiac output, elasticity of the large arteries and blood viscosity. ${ }^{[4]}$ Accordingly, factors affecting the volume of blood in the circulation, the cardiac activity and the compliance and radius of arteries would determine the force that will be exerted on the wall of the arteries.

\section{Hemodynamic Responses to Exercise}

Exercise is a load exerted on the body that the integrative work of the systems of the organism need to adapt. The bodily response for exercise or physical stress is linked with increased metabolic demand which in turn is associated with increased oxygen consumption and blood flow towards the metabolic tissues. In essence, there will be appropriate and

\section{Copyright}

(C) 2020 Phcog.Net. This is an openaccess article distributed under the terms of the Creative Commons Attribution 4.0 International license.

Cite this article: Getu AA. Exercise-Induced Hypertension: A Review of Plausible Mechanisms and Clinical Significance. Int J Clin Exp Physiol. 2020;7(2):48-51. 
often adaptive reactions in the cardiovascular system, respiratory system, the endocrine systems, the renal and the gastrointestinal system. During exercise SBP increases to satisfy the increased demand for oxygenation. Nevertheless, in certain people an abnormal increase in BP is observed and excessive SBP reaction to exercise was shown to be related to future cardiovascular events. ${ }^{[5]}$

Throughout exercise the blood flow to the skeletal muscle will increase, while the flow to the renal and gastrointestinal system will decrease. ${ }^{[6]}$ The cardiac output and minute ventilation would also be increased to cope with increased metabolic demand from the exercising tissues. ${ }^{[7]}$ An increase in blood flow to the skeletal muscles means that there is a profound vasodilation of vasculatures which in turn is expected to decrease the total peripheral resistance. Physical exercise is believed to increase the cardiac sympathetic activity along with a reduction in parasympathetic flow which ultimately increasing the heart rate, stroke volume and hence cardiac output. The enhanced activity of the sympathetic nervous system during exercise also goes to the renal and splanchnic vasculatures and facilitate the redistribution of blood flow from these regions towards the exercising muscles. ${ }^{[8]}$

The influence of the autonomic nervous system on the cardiovascular system during exercise is well recognized. A decreased in vagal activity to the heart is believed to be responsible for the rise in heart rate during initial phases of exercise. However, as the exercise continues, further increase in heart rate is a result of increased in sympathetic activity to the heart which in turn increases the force of contraction and conduction velocity and cardiac output. ${ }^{[9]}$ Reports also showed that the changes occurring in the cardiovascular system during exercise and consequent alterations in blood flow to the skeletal muscles are accompanied by alterations within the parasympathetic and sympathetic nervous system. ${ }^{[10]}$

\section{Exaggerated Blood Pressure Response to Exercise}

The mechanisms underlying an excessive increase in blood pressure during exercise are not well recognized. Risk factors for the development of hypertension are multiple and early detection in otherwise healthy individual is paramount for prevention of the occurrence of hypertension and its complications. This review presents the putative mechanisms proposed to bring an exaggerated blood pressure response to exercise or exercise induced hypertension.

\section{What is Exaggerated Blood Pressure Response to Exercise?}

There is no a single and concise definition to exaggerated blood pressure response to exercise. Some investigators take only elevated systolic $\mathrm{BP}^{[11,12]}$ and others take both systolic and diastolic BPs. ${ }^{[13]}$ Nevertheless, exaggerated blood pressure (hypertensive response) to exercise (treadmill or bicycle) is often defined as a SBP of $\geq 210 \mathrm{mmHg}$ for male and $\geq 190$ for females or a delayed return of SBP to pre-exercise levels or a $10 \mathrm{mmHg}$ increase in DBP from the pre-testing level. ${ }^{[14,15]}$ Studies reported that exercise testing for the presence of hypertensive response to exercise serves as a better risk predictor than resting blood pressure for occurrence of future hypertension and end-organ insults. ${ }^{[1,12]}$

Hypertension during exercise in otherwise normotensive adults is not uncommon. According to several reports, the prevalence ranges from $4 \%$ to $40 \%{ }^{[16]}$ This wide variation in prevalence might be due to the difference in degree of exercise, type of the test (treadmill, Bicycle), the cutoff point for exaggerated blood pressure and the sample size to mention a few. A study done by ${ }^{[13]}$ in normotensive men and women reported that an exaggerated DBP response to exercise was associated with a 2- to 4-fold risk for new-onset hypertension. This study also found that the incidence of hypertension would be substantially high in individuals with a diminished recovery SBP response. A 17 years of follow up study of 4907 otherwise health middle aged men with mildly elevated causal blood pressure by Filipovsky and colleagues indicated that maximal exercise blood pressure is an independent risk factor for cardiovascular diseases. ${ }^{[17]}$

According to Wilson MF, et al. normotensive individuals at risk for the development of hypertension have shown an exaggerated blood pressure response to exercise and this might be due to weak adaptation of the peripheral vasculature in proportion to the cardiac output increased. ${ }^{[12]}$ In summary, exaggerated blood pressure response to exercise can be considered as a disease marker for cardiovascular diseases. Intensive life style modification including diet management can decrease the incidence of exaggerated blood pressure exercise and this can be one reason to consider exaggerated blood pressure response during exercise is a cardiovascular disease marker. ${ }^{[18]}$

\section{Putative Mechanisms}

\section{Neurohormonal Mechanism}

During exercise there will be a reduction of the activity of the parasympathetic nervous system associated with enhanced activity of the sympathetic nervous system. The end results of these often opposite neural entity are an increased heart rate, increased the contractility and stroke volume which ultimately increase cardiac output and hence blood pressure. Additionally, increased sympathetic activity towards vasculatures causes vasoconstriction which in turn increases vascular resistance. An increase in cardiac output together with increased vascular resistance will increase blood pressure. The autonomic nervous system and renin-angiotensin-aldosterone systems play an important role in control of blood pressure during exercise. These systems coordinate together to regulate the function of the cardiovascular system at rest and stressful situation like during exercise.

Studies outlined that stimulation of the sympathetic nervous system and activation of the renin-angiotensin system has been linked with an exaggerated blood pressure response to exercise. ${ }^{[19,20]}$ Abnormal functioning of the autonomic nervous system might be responsible for the pathogenesis of early stages of hypertension. Under normal conditions there is a decline in sympathetic tone and rebound of vagal stimulation during post-exercise period. However, if there is abnormal functioning of the autonomic nervous system to control the heart and blood vessels there might be no proper decline in peripheral resistance which could be one explanation for elevated systolic blood pressure during exercise. ${ }^{[13]}$

There are also evidences supporting the role of angiotensin-II in exaggerated response to exercise. Warner, et al. reported that blocking angiotensin II receptors reduces the peak systolic blood pressure during exercise in individuals with exaggerated blood pressure response to exercise. ${ }^{[21]}$ Chi Young Shim et al. suggested that one putative mechanism for the hypertensive response to exercise is an augmented production of angiotensin II during exercise. ${ }^{[20]}$ These observations indicated the role of angiotensin-II in contributing to an exaggerated blood pressure response to exercise.

\section{A Defect in Arterial Compliance}

The arterial wall is constantly exposed for a force of fluid. The pressure created by this wall stress is determined by the elasticity or compliance nature of the arteries. The wall of arteries especially the larger ones lose compliancy over time. This phenomenon is known as arterial stiffness. Vlachopoulos $\mathrm{C}$ and colleagues speculated that increasing large arterial stiffness is associated with elevated arterial blood pressure and is an independent predictor of mortality under resting conditions. ${ }^{[22]}$ As age increases the elasticity nature of arterial wall is become decreasing 
which might be a risk factor for hypertension and other cardiovascular diseases. Interestingly, life style modifications like $\operatorname{diet}^{[23]}$ and exercise ${ }^{[24]}$ can decrease the degree and progression of arterial stiffness and reduce the incidence of cardiovascular morbidity and mortality.

Laurent $\mathrm{S}$, et al. also reported that one putative factor for an exaggerated response to exercise could be the stiffing of larger arteries which is profoundly presenting with aging and with other concomitant vascular diseases. ${ }^{[25]}$ Additionally, a comparative study done by ${ }^{[26]}$ reported that a reduced arterial compliance in women along with sympathetic activation and increased ventricular filling pressure has an exaggerated blood pressure for exercise testing. This reduction in aortic compliance will reduce the buffering capacity of blood pressure and may result in an abnormal increase in systolic blood pressure.

\section{Impaired Endothelial Function}

The endothelium is a single layer of cells that lines the tunica intima of blood vessels and plays a major role in the modulation of vascular angiogenesis, inflammatory responses and vascular tone and permeability ${ }^{[27]}$ Endothelial cells normally regulate vascular tone by releasing relaxing and constricting factors such as nitric oxide synthase (NOS)-derived nitric oxide (NO), arachidonic acid metabolites, reactive oxygen species (ROS) and vasoactive agents. Exercise induces the vasodilation of blood vessels supplying the exercising muscle tissue thereby increasing blood flow to supply more oxygen and nutrients. Green DJ, et al. reported that as the blood flow increases through arterial vessels hemodynamic drag forces on the endothelium increase and such increases in 'shear' stress stimulate NO release from the endothelium. [28] Adequate and continuous production of $\mathrm{NO}$ is also critical to regulate blood flow and pressure as it has the capacity to counteract the vasoconstrictor tone of sympathetic nervous system. ${ }^{[29,30]}$

Several studies have shown that impairment in endothelial function may be associated with an exaggerated blood pressure response to exercise. ${ }^{[31,32]}$ Tzemos N, et al. found that an exaggerated SBP response to submaximal exercise is closely related to underlying systemic endothelial dysfunction as evidenced by reduced NO bioactivity, reduced proximal aortic compliance and high exercise-induced angiotensin II levels in otherwise healthy sedentary volunteers. ${ }^{[5]}$ Taken together, impairment in the endothelium to play its vasodilator function can be a mediator of exercise induced hypertension and this may be a future study target to quest a preventive and management strategy as exaggerated blood pressure response is a marker of progression to hypertension.

\section{Metabolic Syndrome}

Metabolic syndrome is defined by a group of risk factors that increase the risk of cardiovascular diseases, diabetes and stroke. These risk factors include high blood pressure, abdominal obesity, insulin resistance, dyslipidemia and more. Gaudreault, $\mathrm{V}$ et al. reported that the prevalence of exercise induced hypertension in individuals with metabolic syndrome was $47 \% .{ }^{[33]}$ In their report patients with exaggerated hypertensive response to exercise showed in deterioration in their risk factors. Their anthropometric parameters were increased. Moreover, patients presented with severe metabolic abnormalities including higher low density lipoprotein, fasting blood glucose, as well as insulin resistance. ${ }^{[33]}$ A decrease in insulin sensitivity of tissues found in metabolic syndrome may be involved in the pathogenesis of exercise induced hypertension as studies speculated that insulin resistance is associated with endothelial dysfunction. Evidence also showed that the presence of insulin resistance is associated with activation of both the renin-angiotensin and sympathetic nervous systems which contribute for exaggerated response of blood pressure to exercise. ${ }^{[34]}$
Brett et al. reported that low density lipoprotein cholesterol and insulin resistance have shown to be correlated with increased diastolic blood pressure during maximal exercise test in those study participants without metabolic syndrome. ${ }^{[35]}$ They suggested that low density lipoprotein cholesterol is strongly associated with endothelial malfunction. Thus, this could be involved in exercise induced hypertension in patients with metabolic syndrome by impairing normal endothelial role in vasodilation. ${ }^{[35,36]}$

\section{Conclusion and Future Perspectives}

Exaggerated blood pressure response in otherwise healthy normotensive individuals to exercise is found to be an independent risk factor for the development of hypertension and its target organ complications including variety of cardiovascular diseases. An exaggerated blood pressure response to exercise is associated with a 2 to 3 fold increase in risk of progressive development of hypertension in normotensives. Though there is no a single conclusive explanation as to how exercise induces hypertension arises, evidences showed that the causes are multifactorial. It has been suggested that exaggerated blood pressure response to exercise is related with a failure of total peripheral resistance to reduce during exercise along with hyperactivity of the sympathetic nervous system and the stimulation of angiotensin-II production during exercise. Nevertheless, studies recommend the practice of assessing blood pressure of individuals outside the clinic by utilizing treadmill and bicycle ergometer protocols. Further studies is warranted to identify the mechanism of exercise induced hypertension at the cellular and molecular levels to foster the opportunity to detect abnormalities at earlier times and prevent the progression towards hypertension and its end-organ sequels.

\section{CONFLICT OF INTEREST}

The authors declare no conflict of interest, financial or otherwise.

\section{ABBREVIATIONS}

HRE: Hypertensive Response to Exercise; BP: Blood Pressure; SBP: Systolic Blood Pressure; DBP: Diastolic Blood Pressure; NOS: Nitric Oxide Synthase; ROS: Reactive Oxygen Species.

\section{REFERENCES}

1. Chobanian AV, Bakris GL, Black HR, Cushman WC, Green LA, Izzo JL, et al. Seventh report of the Joint National Committee on Prevention, Detection, Evaluation and Treatment of High Blood Pressure. Hypertension. 2003;42:120652.

2. Kokubo Y, Iwashima Y. Higher Blood Pressure as a Risk Factor for Diseases Other Than Stroke and Ischemic Heart Disease. Hypertension. 2015;66(2):254 9

3. Mills KT, Stefanescu A, He J. The global epidemiology of hypertension. Nat Rev Nephrol. 2020;16:223-37.

4. Barral JP, Croibier A. Visceral Vascular Manipulations. In: Circulatory Physiology. Edinburgh: Churchill Livingstone Elsevier. 2011;27-45.

5. Tzemos N, Lim PO, Mackenzie IS, MacdonaldTM. Exaggerated Exercise Blood Pressure Response and Future Cardiovascular Disease. J Clin Hypertens. 2015;17(11):837-44

6. Pricher MP, Holowatz LA, Williams JT, Lockwood JM, Halliwill JR. Regional hemodynamics during postexercise hypotension. I. Splanchnic and renal circulations. J Appl Physiol. 2004;97(6):2065-70.

7. Joyner MJ, Casey DP. Regulation of increased blood flow (Hyperemia) to muscles during exercise: A hierarchy of competing physiological needs. Physiol Rev. 2015;95(2):549-601.

8. Hayashi N, Yamaoka-Endo M, Someya N, Fukuba Y. Blood flow in non-muscle tissues and organs during exercise: Nature of splanchnic and ocular circulation. J Phys Fit Sport Med. 2012;1(2):281-6.

9. Robertson D, Biaggioni I, Burnstock G, Low PA. Primer on the Autonomic Nervous System. Exercise and the Autonomic Nervous System. Elsevier. $2^{\text {nd }}$ Ed. 2004;183-4

10. Fisher JP. Autonomic control of the heart during exercise in humans: Role of skeletal muscle afferents. Exp Physiol. 2014;99(2):300-5 
11. Dlin RA, Hanne N, Silverberg DS, Bar-Or O. Follow-up of normotensive men with exaggerated blood pressure response to exercise. Am Heart J. 1983;106(2):316-20

12. Wilson MF, Sung BH, Pincomb GA, Lovallo WR. Exaggerated pressure response to exercise in men at risk for systemic hypertension. Am J Cardiol. 1990;66(7):731-6.

13. Singh JP, Larson MG, Manolio TA, O'Donnell CJ, Lauer M, Evans JC, et al. Blood pressure response during treadmill testing as a risk factor for new-onset hypertension. The Framingham heart study. Circulation. 1999;99(14):1831-6.

14. Fletcher GF, Ades PA, Kligfield P, Arena R, Balady GJ, Bittner VA, et al. Exercise standards for testing and training: A scientific statement from the American heart association. Circulation. 2013;128(8):873-934.

15. Schultz MG, La GA, Sharman JE. Blood Pressure Response to Exercise and Cardiovascular Disease. Curr Hypertens Rep. 2017;19(11):1-7.

16. Jae SY, Fernhall B, Lee M, Heffernan KS, Lee MK, Choi YH, et al. Exaggerated blood pressure response to exercise is associated with inflammatory markers. J Cardiopulm Rehabil. 2006;26(3):145-9

17. Filipovsky J, Ducimetiere P, Safar ME. Prognostic significance of exercise blood pressure and heart rate in middle-aged men. Hypertension. 1992;20(3):333-9.

18. Michishita R, Ohta $M$, Ikeda $M$, Jiang $Y$, Yamato $H$. Effects of Lifestyle Modification on an Exaggerated Blood Pressure Response to Exercise in Normotensive Females. Am J Hypertens. 2017:30(10):999-1007.

19. Miyai N, Arita M, Morioka I, Takeda S, Miyashita K. Ambulatory blood pressure, sympathetic activity and left ventricular structure and function in middle-aged normotensive men with exaggerated blood pressure response to exercise. Med Sci Monit. 2005;11(10):478-84.

20. Shim CY, Ha JW, Park S, Choi EY, Choi D, Rim SJ, et al. Exaggerated Blood Pressure Response to Exercise is Associated with Augmented Rise of Angiotensin II During Exercise. J Am Coll Cardiol. 2008;52(4):287-92.

21. Warner JG, Metzger DC, Kitzman DW, Wesley DJ, Little WC. Losartan improves exercise tolerance in patients with diastolic dysfunction and a hypertensive response to exercise. J Am Coll Cardiol. 1999;33(6):1567-72.

22. Vlachopoulos C, Aznaouridis K, Stefanadis C. Prediction of Cardiovascular Events and All-Cause Mortality with Arterial Stiffness: A Systematic Review and Meta-Analysis. J Am Coll Cardiol. 2010;55(13):1318-27.

23. Weisbrod RM, Shiang T, AlSayah L, Fry JL, Bajpai S, Reinhart-King CA, et al. Arterial stiffening precedes systolic hypertension in diet-induced obesity.
Hypertension. 2013;62(6):1105-10

24. Hawkins M, Gabriel KP, Cooper J, Storti KL, Sutton-Tyrrell K, Kriska A. The impact of change in physical activity on change in arterial stiffness in overweight or obese sedentary young adults. Vasc Med. 2014;19(4):257-63.

25. Laurent S, Cockcroft J, Bortel LV, Boutouyrie P, Giannattasio C, Hayoz D, et al. Expert consensus document on arterial stiffness: Methodological issues and clinical applications. Eur Heart J. 2006;27(21):2588-605.

26. Chung H, Kim JY, Lee BK, Min PK, Yoon YW, Hong BK, et al. Arterial stiffness, sex and age difference on hypertensive response to supine bicycle exercise. $J$ Clin Hypertens. 2017;19(12):1260-8.

27. Félétou M, Vanhoutte PM. Endothelial dysfunction: A multifaceted disorder. Am J Physiol Heart Circ Physiol. 2006;291(3):H985-1002.

28. Green DJ, Maiorana A, O'Driscoll G, Taylor R. Effect of exercise training on endothelium-derived nitric oxide function in humans. J Physiol. 2004;56(1):1-25

29. Vallance P, Collier J, Moncada S. Effects of Endothelium-Derived Nitric Oxide on Peripheral Arteriolar Tone in Man. Lancet. 1989;334(8670):997-1000.

30. Stamler JS, Loh E, Roddy MA, Currie KE, Creager MA. Nitric oxide regulates basal systemic and pulmonary vascular resistance in healthy humans. Circulation. 1994;89(5):2035-40.

31. Tzemos N. Is exercise blood pressure a marker of vascular endothelial function?. QJM. 2002;95(7):423-9

32. Stewart KJ, Sung J, Silber HA, Fleg JL, Kelemen MD, Turner KL, et al Exaggerated exercise blood pressure is related to impaired endothelial vasodilator function. Am J Hypertens. 2004;17(4):314-20.

33. Gaudreault V, Després JP, Rhéaume C, Alméras N, Bergeron J, Tremblay A, et al. Exercise-induced hypertension in men with metabolic syndrome: Anthropometric, metabolic and hemodynamic features. Metab Syndr Relat Disord. 2013;11(1):7-14

34. Poirier $P$, Lemieux I, Mauriège $P$, Dewailly $E$, Blanchet $C$, Bergeron J, et al Impact of waist circumference on the relationship between blood pressure and insulin: The Quebec health survey. Hypertension. 2005;45(3):363-7.

35. Brett SE, Ritter JM, Chowienczyk PJ. Diastolic blood pressure changes during exercise positively correlate with serum cholesterol and insulin resistance. Circulation. 2000;101(6):611-5.

36. Creager MA, Cooke JP, Mendelsohn ME, Gallagher SJ, Coleman SM, Loscalzo J, et al. Impaired vasodilation of forearm resistance vessels in hypercholesterolemic humans. J Clin Invest. 1990;86(1):228-34

Cite this article: Getu AA. Exercise Induced-Hypertension: A Review of Plausible Mechanisms and Clinical Significance. Int J Clin Exp Physiol. 2020;7(2):48-51. 